DaLITZ PLOT aNalisis OF THE DECAY $\eta \rightarrow \pi^{+} \pi^{-} \pi^{*}$ )

A.M. Cnops ${ }^{* *}$ ) , G. Finocchiaro ${ }^{* *}$ ) and P. Mittner

CERN, Geneva, Switzerland.

J.P. Dufey, B. Gobbi ${ }^{\dagger}$ and M.A. Pouchon

ETH, Zurich, switzerland

A. Mriler

D.Ph.P.E., Saclay, France

\begin{abstract}
The Dalitz plot distribution of a pure sample of 7170 decays $\eta \rightarrow \pi^{+} \pi^{-} \pi^{\circ}$ is corrected for detection efficiency. Several matrix elements developed up to second order terms have been fitted. The slope of a purely real linear matrix element $\mathrm{ME}=1+\alpha\left[\left(3 \mathrm{~T}_{0} / Q\right)-1\right]$ is found to be $\alpha=-0.55 \pm 0.02$. A slight dependence of the $\mathrm{ME}$ on $\left(\mathrm{T}^{+}-\mathrm{T}^{-}\right)^{2}$ seems to be present. The data are compatible with all coefficients of the MC development being real. Current algebra predictions are discussed. Moreover, upper limits for possible contributions of $\Delta I=0$ and $\Delta I=2$ C-violating amplitudes are given.
\end{abstract}

Geneva - 23 January 1968

(To be submitted to Physies Letters)

*) Work supported in part by the Swiss National Seience Foundation. Present addresses:

**) Brookhaven National Laboratory, Upton, New York.

***) Stony Brook State University, Stony Brook, New York.

t) University of Rochester, Rochester, New York. 

DALITZ PLOT ANALYSIS OF THE DRCAY $\left.\eta \rightarrow \pi^{+} \pi^{-} \pi^{\circ *}\right)$

A. M. Cnops $\left.{ }^{* *}\right)$, G. Finocehiaro ${ }^{* *}$ ) and P. Mittner

CERN, Geneva, Switzerland

J.P. Dufey, B. Gobbi ${ }^{t)}$ and MoA. Pouchon

ETH, Zurich, Switzerland

A. vinller

D.Ph.P.E., Saclay, France

We present here the Dalitz plot density distribution of $\mathbf{7 1 7 0}$ decays $\eta \rightarrow \pi^{+} \pi^{-} \pi^{0}$ obtained in a magnet spark chamber experiment. The experimental set-up and the details of the analysis are described in a previous paper ${ }^{1}$ ) where the results on the charge asymetry are presented.

In addition to the criteria used in Ref. 1 to select the sample of $\eta \rightarrow \pi^{+} \pi^{-} \pi^{0}$, the following cuts have been applied:

i) both tracks wust cross the tenth gap inside a square of $60 \times 60 \mathrm{~cm}^{2}$;

ii) the momentum component perpendicular to the magnetic field has to be larger than $80 \mathrm{MeV} / \mathrm{o}$ for each pion;

iii) $M_{e^{+} e^{-}}>10^{-3}\left(\mathrm{GeV} / \mathrm{c}^{2}\right)^{2}$ where $M_{e^{+}}-$is the invariant mass of the two charged particles assumed to be electrons. This cut removes most of the Dalitz pairs.

The events which failed in the pattern recognition and geometry programme have been remeasured on digitized projectors. About 300 of these remeasurements passed the selection criteria and have been added to the final sample.

The sample of $7170 \eta$ decays is nearly free of background; there remains (Ref. 1) less than $1 \%$ of non-eta events, and about $1 \%$ of contamination due to the decay mode $\eta \rightarrow \pi^{+} \pi^{-} \gamma$. In order to study the Dalitz plot density distribution, it is necessary to compute the detection efficiencies for each region

*) Work supported in part by the Swiss National Science Foundation. Present addresses:

**) Brookhaven National Laboratory, Upt on, New York.

***) Stony Brook State University, Stony Brook, New York.

t) University of Rochester, Rochester, New York. 
of the Dalitz plot. This has been done by means of a high statistics Monte Carlo calculation, which simulates in detail the geometry of the apparatus and takes into account effects such as the pion decays in flight, the rescattering probability, and all the geometrical and kinematical cuts. Moreover, the time-of-flight spectra of the neutrons for the accepted Monte Carlo events reproduce the experimental spectra, and the number of events associated with each neutron counter is proportional to the corresponding experimental number. It must be noted that the geometrical cuts eliminate those events whose detection efficiencies are not easily computable due to strong curvatures of the tracks, large angles with the normal to the plates, or inefficiency of the spark chambers on the edges.

The efficiency is found to vary by a factor of 4 along the $y$ axis and by a factor of 1.4 al ong the $x$ axis of the Dalitz plot. The subtraction of the $1 \% \eta \rightarrow \pi^{+} \pi^{-} \gamma$ background is performed by using a sample of Monte Carlo generated $\pi^{+} \pi^{-} \gamma^{\prime} s$, simulating in detail (up to the Coulomb scattering and the spark fluctuations al ong the trajectories) the real events. Those which survived the normal chain of programmes and the criteria defining the $\pi^{+} \pi^{-} \pi^{0}$ sample were subtracted after proper normalization.

A general check of our method of analysis is obtained by comparing, for several laboratory quantities (angles, momenta, etc.) the distributions relative to the real and the Monte Carlo $\eta \rightarrow \pi^{+} \pi^{-} \pi^{0}$ events (a linear matrix element is used in the generation). A good consistency was found for each pair of corresponding distributions. The coordinates on the Dalitz plot are defined as: $\quad x=\sqrt{3}\left(T^{+}-T^{-}\right) / Q, y=3 T^{0} / Q-1$, where: $Q=M_{\eta}-M \pi^{+}-M_{\pi^{-}}^{-} M_{\pi^{0}}$ and $T^{0,+,-}$ are the kinetic energies of the $\pi^{0,+},-$ in the $\eta$ rest frame.

A first fit is performed on the corrected distribution $\mathrm{dN} / \mathrm{dy}$ of the numbers of events (projected on the $y$ axis) with the expression: $F(y)=\Phi(y) \cdot|M|^{2}$, where $\Phi(y)$ is the phase space and $M$ is a linear matrix element: $M=1+\alpha \cdot y$ (24 bins are used). We find: $\alpha=-0.557 \pm 0.013$ with a $\chi^{2}$ of 23.6 for 22 degrees of freedom. In this approximation, the matrix element is therefore compatible with linearity in $\mathrm{y}$ and $\alpha$ real.

We can now obtain an over-all check of internal consistency of our data by computing a for several independent samples of events (which have in general different efficiencies). Each sample is defined by means of the gap interval 

where the shortest track comes out of the fiducial volume. The results are displayed in Table 1. The compatibility is good and this test gives no evidence for systematic errors. However, systematic errors of the same order as the statistical ones cannot be excluded.

Table 1

\begin{tabular}{|c|c|c|}
\hline$\alpha$ & $\begin{array}{c}\chi^{2} \text { for 22 degrees } \\
\text { of freedom }\end{array}$ & Gap \\
\hline$-0.548 \pm 0.029$ & 25 & $10-12$ \\
$-0.522 \pm 0.043$ & 46 & $12-14$ \\
$-0.572 \pm 0.029$ & 16 & $14-16$ \\
$-0.539 \pm 0.039$ & 21 & $16-18$ \\
$-0.504 \pm 0.052$ & 27 & $18-20$ \\
$-0.593 \pm 0.061$ & 28 & $20-22$ \\
$-0.614 \pm 0.031$ & 18 & $>22$ \\
\hline
\end{tabular}

In the subsequent analysis we use a more detailed description of the Dalitz plot. The ranges of variation of both $y$ and $x$ are divided into twelve intervals each, and each box is corrected for the background and the efficiency (Fig. 1).

\section{C invariance}

C-conserving matrix elements are symmetric in $x$. A left-right asymmetry would indicate an interference with a $\mathrm{C}$ non-conserving amplitude. The asymetry published in Ref. 1 was found to be $0.3 \pm 1.1 \%$ for a larger sample of 10,665 events. In the present sample a matrix element of the following form has been fitted: 


$$
M(x, y) \sim 1+\alpha y+\beta y^{2}+\gamma x^{2}+\delta x
$$

The fitted value for $\delta$ is $\delta=0.006 \pm 0.013$ with $\chi^{2}=115.9$ for 116 degrees of freedom. $\delta$ compatible with 0 indicates that there is no evidence for a $T=2$ amplitude.

The following matrix element has been fitted in order to investigate the presence of a $T=0$ amplitude:

$$
M(x, y) \sim 1+\alpha y+\beta y^{2}+\gamma x^{2}+\delta\left(3 y^{2}-x^{2}\right) x .
$$

$\left(3 y^{2}-x^{2}\right) x$ is the simplest matrix element for the $T=0$ state. The result is $\delta=0.00 \pm 0.02, \chi^{2}=116.1$ for 116 degrees of freedom.

\section{C-invariant matrix el ement}

The most general C-invariant matrix element can be written ${ }^{2}$ ) (up to the third order):

$$
\mathrm{M}=1+b \mathrm{Y}^{0}+c \mathrm{Y}^{0^{2}}+\mathrm{dY}^{+} \mathrm{Y}^{-}+e \mathrm{Y}^{03}+f \mathrm{Y}^{+} \mathrm{Y}^{-} \mathrm{Y}^{0}
$$

with

$$
\mathrm{Y}^{0,+,-}=\frac{3 \mathrm{~T}^{0,+,-}}{Q}-1
$$

The coefficients are complex numbers. The fit has been performed on the folded Dalitz plot, the matrix element being symmetrio by exchanging $\mathrm{Y}^{+}$into $\mathrm{Y}^{-}$.

\section{First order fit:}

$$
\mathbf{u}=1+\left(b_{i}+i b_{z}\right) y^{0}
$$

The fitted values are:

$$
\begin{aligned}
& b_{1}=-0.550 \pm 0.012 \\
& b_{2}=0.00 \pm 0.11 \\
& x^{2}=67.4 \text { for } 58 \text { degrees of freedom. }
\end{aligned}
$$

Second order fit

$$
M=1+\left(b_{1}+i b_{2}\right) Y^{0}+\left(c_{1}+i c_{2}\right) Y^{0^{2}}+\left(d_{1}+i d_{2}\right) Y^{+} Y^{-}
$$


which gives the result:

$$
\begin{aligned}
& b_{1}=-0.567+0.031 \quad b_{2}=0.482+0.33 \\
& \begin{array}{ll}
-0.031 & -0.50
\end{array} \\
& c_{1}=-0.177 \begin{array}{c}
+0.140 \\
-0.370
\end{array} \quad c_{2}=0.053+0.61 \\
& d_{1}=-0.101+0.046 \quad d_{2}=0.110+0.36
\end{aligned}
$$

The $\chi^{2}$ indicates that it is not necessary to fit a higher order matrix element.

The second order fit has also been performed neglecting the imaginary parts。 Then:

$$
\begin{aligned}
& b=-0.545 \pm 0.015 \\
& c=-0.026 \pm 0.034 \\
& d=-0.082 \pm 0.040 \\
& \chi^{2}=58.3 \text { with } 57 \text { degrees of freedom. }
\end{aligned}
$$

The projection of the fitted $|M|^{2}$ on the $Y^{0}$ axis together with the experimental point is plotted in Fig. 2.

In conclugion, the gecond order terme are gufficient to degerihe our data. The results are compatible with the coefficients $b$ and $d$ being purely real and no quadratic terin in $Y^{0}$. However, a slight dependence on $Y^{+} \cdot Y^{-}$, or $x^{2}$, seems to be required [notice that: $X^{2}=\left(Y^{0^{2}}-4 Y^{+} Y^{-}\right) / 3$ ]. In the approximation in which the Watson theorem is valid, an absence of imaginary terms (i.e. no variation of the phase of the matrix element within the energy range considered) could be interpreted ${ }^{3}$ ) as an indication in favour of a relatively weak $\pi^{+} \pi^{-}$interaction ${ }^{4}$ ) Although our data can be fitted using only real paraneters, we cannot exclude the existence of a relatively large imaginary part, especially if the matrix element is not assumed to be linear. 

B.W. Lee parametrization of the matrix element ${ }^{5}$ )

$M$ is expanded as a sum of orthonormal functions of two new parameters $\alpha$ and $\beta$, related to $\rho$ and $\theta$, the polar coordinates with respect to the centre of the Dalitz plot:

$$
\begin{aligned}
& \alpha=\rho(\mathrm{x}, \mathrm{y}) / \rho_{\max }(\vartheta) \\
& \beta(\vartheta)=\mathrm{K} \cdot \int_{0}^{\vartheta} \sigma_{\max }^{2}\left(\vartheta^{\prime}\right) \mathrm{d} \vartheta^{\prime}
\end{aligned}
$$

with $K$ fixed by the condition $\beta(\pi)=\pi$.

Then

$$
M=\sum_{i} a_{i} \cdot F_{i}(\alpha, \beta)
$$

with

$$
\begin{aligned}
& F_{1}=1 / \sqrt{\pi} \\
& F_{2}=2 / \sqrt{\pi} \alpha \cdot \cos \beta \\
& F_{3}=\sqrt{3 / \pi}\left(2 \alpha^{2}-1\right) \\
& F_{4}=\sqrt{6 / \pi} \alpha^{2} \cdot \cos 2 \beta
\end{aligned}
$$

The fit to the experimental data of $M=F_{1}+a_{2} \cdot F_{2}$ gives:

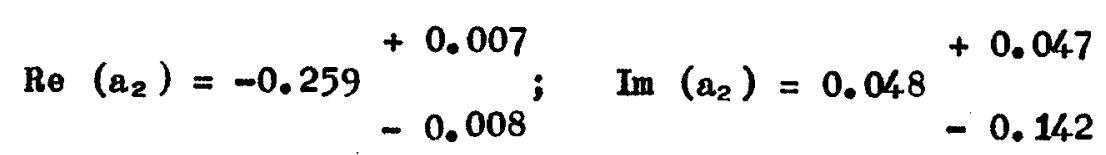

with a $\chi^{2}$ of 58.5 for 58 degrees of freodom. If only Re $\left(a_{2}\right)$ is used we obtain:

$$
\operatorname{Re}\left(a_{2}\right)=-0.258 \pm 0.006,
$$


with $\chi^{2}=58.6$ for 59 degrees of freedome At the present level of statistics with this parametrization, a real matrix element with a single parameter is therefore sufficient to describe the whole Dalitz plot density distribution ${ }^{*}$. The $y$ projection of $|M|^{2}$ is plotted in Fig. 2.

\section{Current Algebra}

It has been shown by several authors ${ }^{6}$ ) that current algebra (CA) requires the matrix element (MEI) to be zero at the three points outside the physical region where $q_{\mu}$, the four momentum of either pion, is zero. These points cannot be represented on the Dalitz plot (where the condition $q^{2}=m_{\pi}^{2}$ is verified); however, if we believe that the dependence of the $M E$ on $\mathrm{q}^{2}$ is negligible, the CA prescriptions should be valid on the Dalitz plot, at the three points defined by:

$$
\begin{aligned}
& \left(\mathbb{E}^{ \pm}=0, \mathbb{E}^{0}=\mathbb{E}^{\overline{+}}=1 / 2 \mathrm{M} \eta\right), \\
& \left(\mathbb{E}^{0}=0,{E^{+}}^{+}=E^{-}=1 / 2 M \eta\right),
\end{aligned}
$$

$E^{+,-, 0}$ being the total energies of the pions (for the kinetic energies, $\mathrm{E}^{+,-, 0}=0$ corresponds to $\mathrm{T}^{+,-, 0}=-\mathrm{m}, \pi^{+,-, 0}$ ). It is therefore important to see whether the simplest $M E$ which $\mathbb{f}_{i t}$ our data inside the physical region are conpatible with 0 , when extrapolated to these three points.

This condition comes out to be verified (for both parametrizations used) at the points where $\left(\mathrm{T}^{ \pm}=-\mathrm{m}_{\pi}, \mathrm{T}^{\circ} \approx \mathrm{m}_{\pi}\right)$, but it does not work at $\mathrm{T}^{\circ}=-\mathrm{m}_{\pi^{\circ}}$

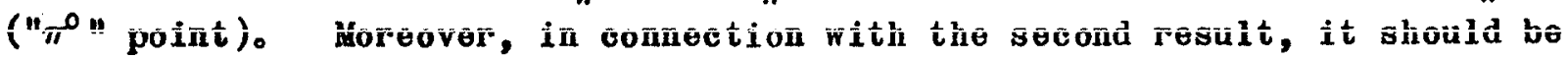
noted that our data do not give any real indication in favour of a "bending"

*) A fit with the Brown and Singer model (s-wave dipion resonance) gives a $\chi^{2}$ of 97.7 for 58 degrees of freedom. The best fitted values for the mass and width are, respectively:

$$
\mathrm{M}=394 \mathrm{MeV}, \quad \Gamma=92 \mathrm{MeV} \text { 。 }
$$

In order to check the consistency of the data we have also made a fit, without using the two boxes corresponding to the lowest $T^{\circ}$ value. Then: $M=389 \mathrm{MeV}, \Gamma=85.5 \mathrm{MeV}, \chi^{2}=76.7$ for 56 degrees of freedom. 
of the in the direction of the $" n^{0} "$ point (no significant term in $\mathrm{y}^{2}$, or of higher order, is present). One way of overcoming this difficulty consists ${ }^{7}$ ) of admitting also a $q_{0}^{2}$ dependence of the Me (which had previously been neglected). If a linear dependence on $q_{0}^{2}$ is assumed, then the $C A$ constraints can be satisfied by an expression which - on the $\pi^{0}$ mass shell is simply linear in $T^{0}$, and vanishes only at the " $\pi^{ \pm}$points, in agreement with the observed behaviour.

Obviously, it is also possible, by introduoing higher order terms to obtain a reasonable fit to our data, with the supplementary constraint of the $M$ being equal to zero at the three points of interest. In the B.W. Lee parametrization, using terms up to the second order, we get a $\chi^{2}$ of 63.2 on 54 degrees of freedom; the inclusion of the third order terms gives a $\chi^{2}=54.7$, on 50 degrees of freedom.

In conclusion, our results reproduce, at a higher level of precision, the well-known difficulties in the choice of an extrapolation of the ME in the region where Eo $\simeq 0$. On the other hand, the fact that the extrapolation in the direction of the " $\pi^{+}$" points (i.e. beyond a value of the $\pi^{+} \pi^{-}$ effective mass, $M^{+}-=2 m_{\pi^{\prime}}$ ) works well, may be considered as an indication that the threshold $\pi^{+} \pi^{-}$fect in the $\pi^{+} \pi^{-}$channel is weak, again favouring (together with the absence of an imaginary part in the ME) a weak $\pi^{+} \pi^{-}$interaction. All the errors quoted in this work are purely statistical. As already stressed, systematic errors of the order of the statistical ones cannot be excluded. For comparison and practical use of our results we would advise multiplying all errors by 1.5 .

Our final value for the slope of the linear matrix element is then:

$$
\alpha=-0.55 \pm 0.02
$$

The compilation of events of several bubble chamber experiments ${ }^{8}$ ) gives for a the value $-0.48 \pm 0.04$. The result of Larribe et al. 9 ) cannot be directly compared, the fit having been made with $|\mathrm{M}|^{2}$ linear. If we reduce their result by neglecting the quadratic term we obtain $\alpha=-0.472 \pm 0.035$.

In a paper to be published later we will examine which are the simplest matrix elements needed in order to describe both the density distribution of our Dalitz plot, and given values of the branching ratio $\eta \rightarrow 3 \pi^{0} / \eta \rightarrow \pi^{+} \pi^{-} \pi^{0}$. 


\section{Acknow ledgements}

We are very grateful to Prof. P. Preiswerk for his encouragement and support, to Dr. D.G. Sutherland and Dr. M. Veltman for many discussions on the argument, and to Dr. A. Larribe for his important help in the Monte Carlo calculations. 
REEERENCES

1) A.M. Cnops, G. Finocchiaro, J.C. Lassalle, P. Mittner, P. Zanella, J.P. Dufey, B. Gobbi, M.A. Pouchon and A Miller, Physics Letters 22 $546(1966)$.

2) L.R. Price and F.S. Crawford, UCRL - 17629 preprint (1967).

3) We are very grateful to Dr. M. Jacob for several illuminating discussions on this subject.

4) We assume here that the $\pi^{+-} \pi^{-} I=0$ interaction would provide the most prominent final-state interaction. This is in agreement with the fact that the observed dependence of the matrix element on $Y^{+} Y^{-}$is slight, if not negligible [a $Y^{+} Y^{-}$dependence should be interpreted as the effect of a $\pi^{\circ} \pi^{-}(I=1,2)$ interaction].

5) B.W. Lee, Harmonic analysis of the Dalitz plot, CTS-HE-67-3, University of Miami, preprint March 67.

6) D.G. Sutherland, Physics Letters 23, 384 (1966); see also T.S. Bell and D.G. Sutherland, CERN preprint TH 822 (1967), and M. Jacob, CERN preprint TH 81,6 (1967), for other references and discussions on the subject.

7) W.A. Bardeen, Lowell S. Brown, B.W. Lee and H.T. Nick, Phys. Rev. Letters 18, 1170 (1967).

8) Columbia-Berkeley-Perdue-Wisconsin-Yale collaboration, Phys. Rev。 149. 1044 (1966).

9) A. Larribe et al., Saclay-Rutherford collaboration, Physics Letters 23, $600(1966)$. 


\section{Figure captions}

Fig. 1 : Dalitz plot population. In each cell the two numbers correspond to the relative number of events in that cell (corrected for background and efficiency) and to the error on this number.

Fig. 2 : Projection on the $\mathrm{T}^{\circ}$ axis of the squared modulus of the $\mathrm{ME}$ curve $A$ : fitted $M E=1+b Y^{0}+e Y^{0^{2}}+d Y^{+} Y^{-}$with $b, c$, and $d$ real. Curve $B$ : $M E$ in the $B_{0} W$. Lee parametrization with a single real parameter. 


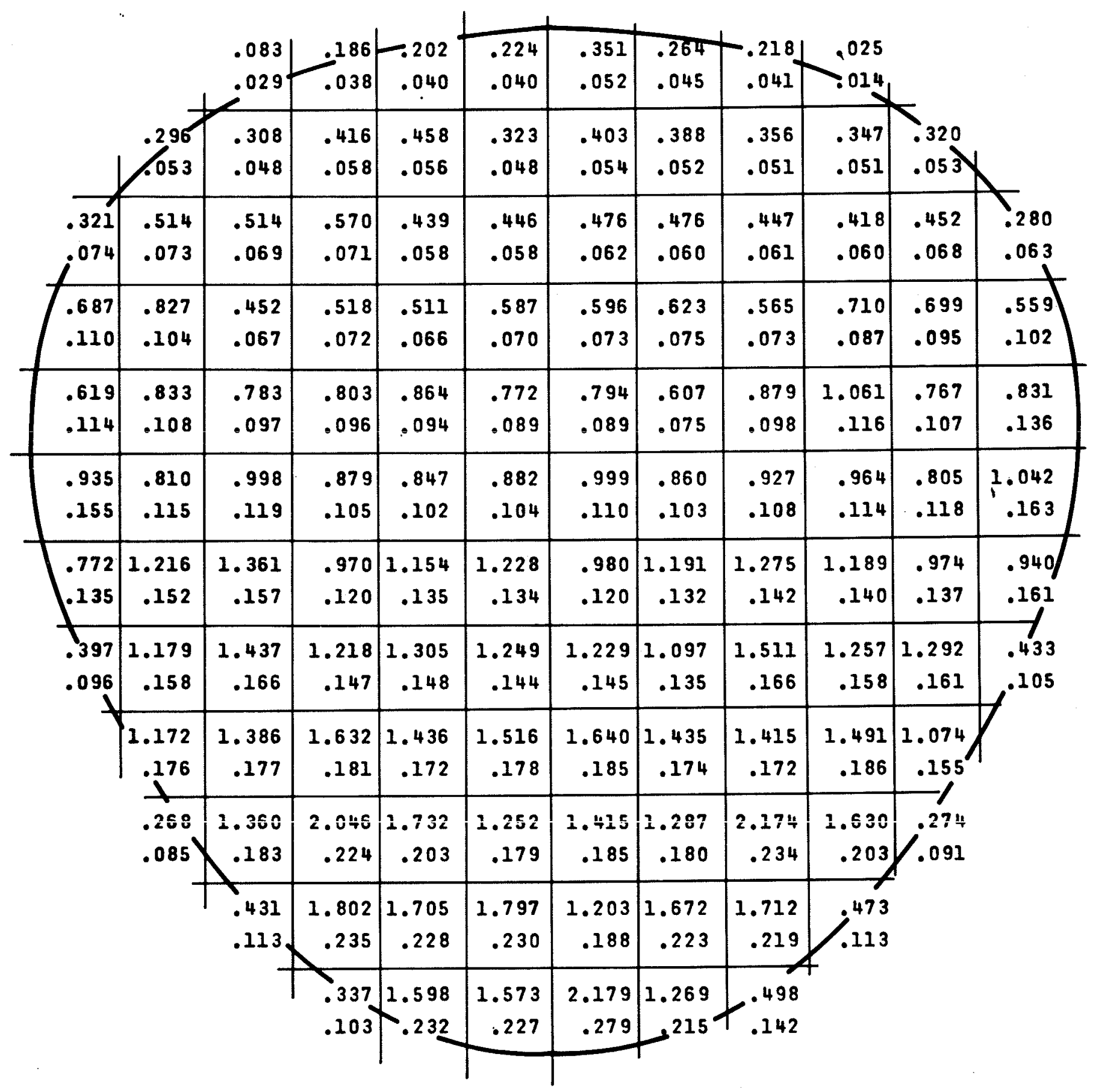




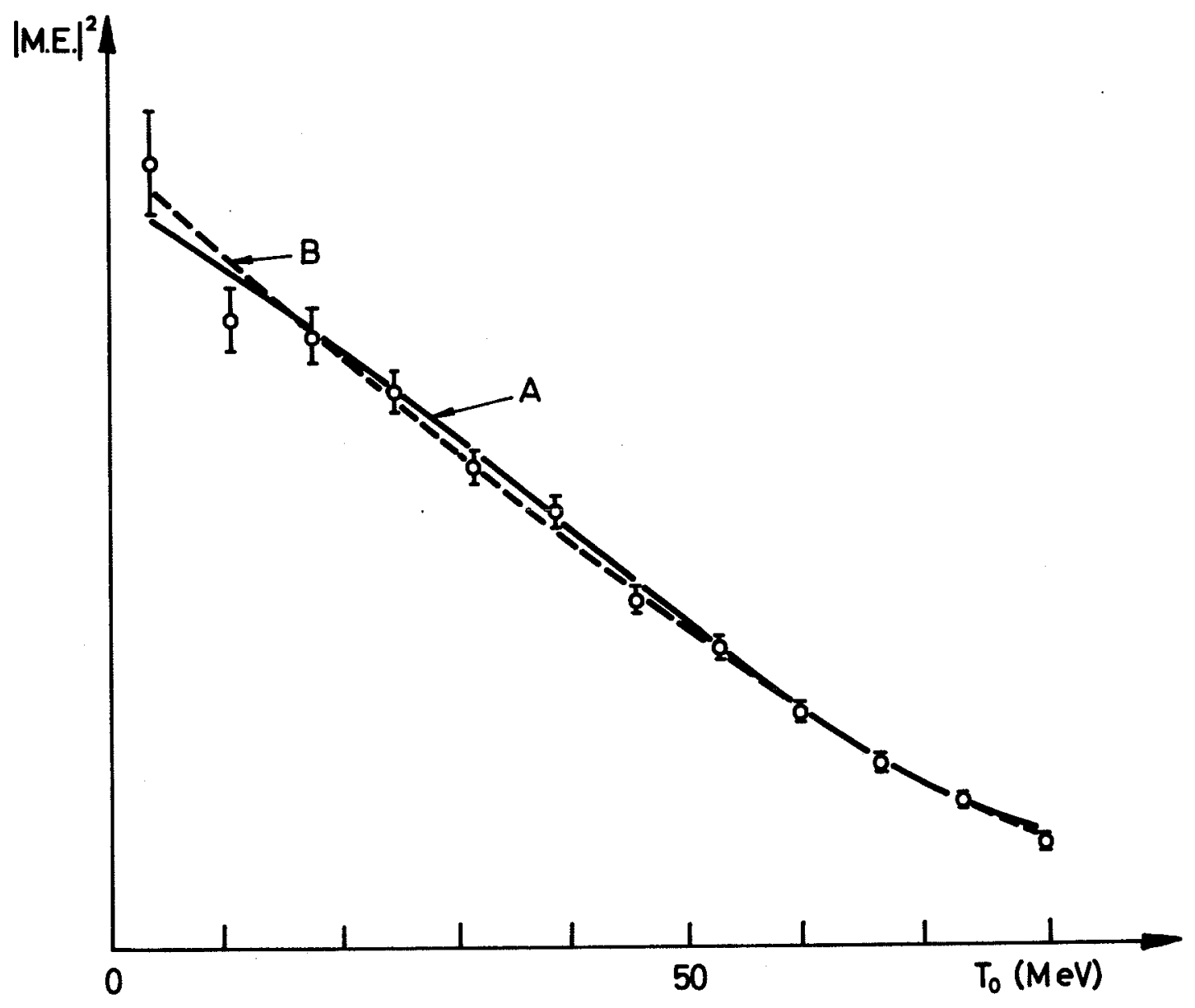

Fis. 2 
